\title{
Criminologie
}

\section{L'intervention auprès de jeunes mères et de leur enfant : perspective de la théorie de l'attachement}

\section{George M. Tarabulsy, Julie Robitaille, Carl Lacharité, Julie Deslandes et Rémi Coderre}

Volume 31, numéro 1, printemps 1998

La prévention de l’inadaptation sociale : approche développementale

URI : https://id.erudit.org/iderudit/017409ar

DOI : https://doi.org/10.7202/017409ar

Aller au sommaire du numéro

Éditeur(s)

Les Presses de l'Université de Montréal

ISSN

0316-0041 (imprimé)

1492-1367 (numérique)

Découvrir la revue

Citer cet article

Tarabulsy, G. M., Robitaille, J., Lacharité, C., Deslandes, J. \& Coderre, R. (1998).

L'intervention auprès de jeunes mères et de leur enfant : perspective de la

théorie de l'attachement. Criminologie, 31(1), 7-23.

https://doi.org/10.7202/017409ar

\section{Résumé de l'article}

The children of adolescent mothers are among those presenting the highest level of psychosocial risk, compromising their socio-emotional development on a number of levels. Several intervention strategies have been designed to address some of the problems that characterize these mother-infant dyads. While these strategies have proven to be beneficial to young mothers, relatively little impact has been found on the level of infant and child development. The purpose of this article is to address the issue of intervention aimed at adolescent mothers and their infant, and underline that the relational dimension, while absent from most intervention programs, is necessary to our understanding of the problems presented by these dyads. An intervention program, currently undergoing evaluation, is described having as a main objective the improvement of the early mother-infant relationship. Attachment theory is presented as an organizing construct. Characteristics of the intervention, as well as the evaluation strategy, are described.
Tous droits réservés ( Les Presses de l'Université de Montréal, 1998
Ce document est protégé par la loi sur le droit d'auteur. L’utilisation des services d'Érudit (y compris la reproduction) est assujettie à sa politique d'utilisation que vous pouvez consulter en ligne.

https://apropos.erudit.org/fr/usagers/politique-dutilisation/ 
L'INTERVENTION AUPRÈS DE JEUNES MÈRES ET DE LEUR ENFANT : PERSPECTIVE DE LA THÉORIE DE L'ATTACHEMENT ${ }^{1,2}$

George M. Tarabulsy, Julie Robitaille, Carl Lacharité, Julie Deslandes et Rémi Coderre ${ }^{3}$

The children of adolescent mothers are among those presenting the highest level of psychosocial risk, compromising their socioemotional development on a number of levels. Several intervention strategies have been designed to address some of the problems that characterize these mother-infant dyads. While these strategies have proven to be beneficial to young mothers, relatively little impact has been found on the level of infant and child development. The purpose of this article is to address the issue of intervention aimed at adolescent mothers and their infant, and underline that the relational dimension, while absent from most intervention programs, is necessary to our understanding of the problems presented by these dyads. An intervention program, currently undergoing evaluation, is described having as a main objective the improvement of the early mother-infant relationship. Attachment theory is presented as an organizing construct. Characteristics of the intervention, as well as the evaluation strategy, are described.

Les travaux de Dukewich, Borkowski et Whitman (1996) aux États Unis, ainsi que ceux de Turner, Grindstaff et Phillips (1990) au Canada, démontrent que le taux de fécondité des adolescentes est en augmentation en Amérique. Au Québec, les naissances chez les adolescentes compte pour $11 \%$ de l'ensemble des naissances. Durant les 15 dernières années, malgré une diminution de la proportion d'adolescents dans la population, le taux de

1. Une version plus détaillée de cet article peut être obtenue en communiquant avec les auteurs.

2. La réalisation de cet article à été rendue possible grâce à une subvention de recherche du Conseil québécois de la recherche sociale et une subvention interne de l'Université du Québec à Trois-Rivières au premier auteur. Nous remercions également les membres du GREDEF pour leur commentaires constructifs.

3. Groupe de recherche en développement de l'enfant et de la famille (GREDEF), Département de psychologie, Université du Québec à Trois-Rivières, C.P. 500, Trois-Rivières (Québec), Canada, G9A 5H7. 
fécondité des adolescentes a augmenté de $22 \%$. Même si les naissances chez les mères de milieux défavorisés ont chuté de $40 \%$ depuis 1981 , les naissances chez les adolescentes défavorisées ont augmenté de $9 \%$ (Pageau, Ferland, Choinère et Sauvageau, 1997).

Les auteurs de plusieurs recensions récentes s'entendent pour dire que, de toutes les clientèles à risque sur le plan psychosocial, les enfants de mères adolescentes sont parmi celles ayant le pronostic développemental le moins favorable (Brooks-Gunn et Furstenberg, 1986 ; Schellenbach, Whitman et Borkowski, 1992 ; Trad, 1994). Il n'est donc pas surprenant que bon nombre de programmes d'intervention ont été développés afin de favoriser le développement des enfants de jeunes mères. Le but de cet article est d'aborder la question de l'intervention auprès des jeunes mères et de leur enfant, en poursuivant quatre objectifs précis : premièrement, nous présenterons les principales stratégies d'intervention telles qu'elles sont actuellement implantées dans divers contextes ; deuxièmement, nous démontrerons l'importance dans cette problématique de la question du vécu relationnel des jeunes mères, pourtant souvent absente de l'élaboration des stratégies d'intervention ; troisièmement, nous proposons l'idée que la théorie de l'attachement s'avère un outil approprié sur le plan scientifique pour aborder la question des relations vécues par les jeunes mères; quatrièmement, nous présentons un programme d'intervention que nous évaluons à Trois-Rivières, portant sur le développement de la relation mère-enfant durant la première année et fondé sur la théorie et les méthodes de l'attachement.

\section{L'INTERVENTION AUPRÈS DES DYADES ENFANT - MÈRE ADOLESCENTE}

La majorité des travaux de recherche sur les enfants de mères adolescentes sont centrés sur la description de leurs contextes de vie. L'objectif implicite de cette démarche est d'identifier les caractéristiques contextuelles à la source des difficultés des jeunes mères. Dans cette perspective, l'identification première de tels facteurs structuraux permet par la suite de les modifier afin d'influencer le bien-être de la jeune mère, sa compétence parentale et, par conséquent, le développement de son enfant (voir Chamberland, 1996). Les résultats de ces travaux ont démontré que l'arrivée d'un enfant dans un foyer où la mère est adolescente est, dans la plupart des cas, accompagnée d'une série de facteurs de risque exerçant un impact sur les plans biologique (Brooks-Gunn et Furstenberg, 1986 ; Trad, 1994), psychologique et social (Brooks-Gunn et Furstenberg, 1987 ; Lamb, 1988). Souvent, les mères adolescentes proviennent de milieux qui se caractérisent par l'isolement, la pauvreté et la dépendance de l'État pour subvenir à leurs besoins essentiels ; elles sont plus susceptibles d'avoir eu des problèmes judiciaires, 
de décrocher de l'école et de vivre des problèmes d'ajustement psychologique - certains travaux démontrant des taux de dépression de l'ordre de $60 \%$ (Osofsky, Culp et Ware, 1988). Elles vivent de nombreuses ruptures de relations et, dans plus de la moitié des cas, la naissance de l'enfant a lieu dans un foyer monoparental (Spieker et Bensley, 1994). De plus, la jeune mère n'a pas la maturité cognitive et émotionnelle nécessaire pour prendre soin de son enfant (Osofsky, Hans et Peebles, 1993). C'est dans cette " niche écologique " que les enfants de mères adolescentes naissent.

Les stratégies d'intervention visant à améliorer les conditions des jeunes mères portent généralement sur l'une des trois facettes suivantes de l'écologie de la dyade : 1) la dimension biomédicale ; 2) l'éducation des jeunes mères concernant le développement de leur enfant ; ou 3) le soutien social. Compte tenu que cette présentation porte principalement sur les dimensions psychologiques et sociales des dyades enfant-mère adolescente, seules les stratégies 2 et 3 sont abordées (voir Trad, 1994, concernant l'intervention biomédicale).

\section{Éducation parentale}

Il est bien documenté que les jeunes mères possèdent des connaissances imprécises du développement de l'enfant, des attitudes parentales inappropriées, ainsi qu'un manque d'information sur les soins à lui donner (Schellenbach et al., 1992). Dans cette optique, on conçoit qu'en augmentant les connaissances des jeunes mères, ces dernières sauront manifester davantage de compétence envers leur enfant. Ces programmes s'effectuent habituellement sous forme de rencontres de groupe ou individuelles où l'on présente différentes informations sur le développement des enfants et la façon d'en prendre soin. Parfois, ces rencontres impliquent l'enfant directement et des exercices pratiques sont abordés afin de donner à la jeune mère des outils favorisant la stimulation mentale et motrice de son enfant. Ces programmes ont une durée variable mais la majorité sont implantés entre le septième mois de grossesse et le deuxième anniversaire de l'enfant (Benasich, Brooks-Gunn et Clewell, 1992).

Ces initiatives comportent trois objectifs. Le premier objectif est direct et concerne l'amélioration des connaissances maternelles du développement. Les deuxième et troisième objectifs sont indirects et portent sur l'amélioration des comportements maternels et du développement de l'enfant. Bien que ces trois objectifs soient stipulés dans l'élaboration de ces programmes d'intervention, l'évaluation de leur efficacité porte principalement sur le premier. À cet égard, plusieurs études démontrent que la participation à de tels programmes améliore chez les jeunes mères les connaissances du développement (Benasich et al., 1992 ; Trad, 1994). 
Peu d'études ont examiné l'impact de telles interventions sur les comportements maternels à l'égard de l'enfant ou sur le développement de l'enfant. Parmi celles qui se sont penchées sur le développement cognitif et moteur, on retrouve régulièrement des effets non significatifs (Benasich $\boldsymbol{e t}$ al., 1992 ; Clewell, Brooks-Gunn et Benasich, 1989). Notons qu'aucune étude répertoriée ne fait l'évaluation de chacune des trois catégories d'objectifs.

On constate également que les études évaluatives présentent régulièrement des limites méthodologiques : des mesures non validées sont souvent utilisées et il est rare de trouver un groupe de comparaison. L'évaluation se limite souvent à des rapports maternels et l'effet est rarement suivi longitudinalement. Force est d'admettre que la recherche auprès des jeunes mères implique souvent de petits échantillons et une attrition importante. Cependant, ces problèmes logistiques, jumelés à des résultats inconstants, indiquent que l'impact des programmes d'éducation parentale se situe surtout au niveau des connaissances et qu'il n'y a que peu d'effets sur les comportements maternels et le développement des enfants (Clewell et al., 1989).

\section{Amélioration du soutien social}

La question du soutien social est particulièrement importante pour les jeunes mères pour deux raisons. Premièrement, les jeunes mères vivent souvent en situation d'isolement et les gens de leur entourage démontrent un plus faible potentiel d'aide que ceux des mères adultes (Spieker et Bensley, 1994). Deuxièmement, contrairement à la situation des mères de classe moyenne, la source de soutien principale des jeunes mères n'est pas le père biologique de l'enfant, le soutien provenant de diverses personnes de leur réseau (Spieker et Bensley, 1994). Dans la majorité des cas, les jeunes mères se retrouvent sans le père de l'enfant peu après l'accouchement (Osofsky et al., 1988). Par ailleurs, il n'est pas clair que sa présence dans l'environnement de la dyade mère-enfant soit en effet un soutien, plusieurs études démontrant même des effets négatifs. Dans cette perspective, la question de l'amélioration du système de soutien devient pertinente.

Encore ici, il y a des objectifs directs et indirects à ces stratégies, l'objectif direct étant d'augmenter la taille et d'améliorer la qualité du réseau social afin de donner accès à un plus grand potentiel d'aide à la jeune mère. Cette stratégie est reconnue pour contribuer à la diminution du stress et de la dépression expérimentée par les nouvelles mères (Osofsky et al., 1988). L'objectif indirect découle du fait que si la jeune mère se sent mieux, moins stressée et mieux soutenue, elle sera plus disponible pour répondre adéquatement aux besoins et signaux de son enfant (Jacobson et Frye, 1991). 
Dans de telles interventions, les jeunes mères sont appelées à participer à des groupes dans lesquels on aborde certains objectifs d'éducation parentale. D'autres thématiques sont également abordées (ex. relations de couples, école), le but principal étant de favoriser les liens entre les jeunes mères, entre les jeunes mères et des mères adultes, ou encore entre les jeunes mères et les animatrices. Certaines stratégies tentent de rehausser le soutien provenant d'un membre de la famille de la jeune mère, souvent sa propre mère ou le père de l'enfant (Spieker et Bensley, 1994). Parfois, le soutien accordé aux mères comporte un objectif instrumental précis, comme l'offre de service de garde pour qu'elle puisse poursuivre ses études (Clewell et al., 1989).

Comme c'est le cas pour la stratégie d'éducation parentale, les résultats obtenus dans les quelques recherches évaluatives menées jusqu'ici indiquent que les effets les plus saillants concernent davantage la mère et moins le développement de l'enfant. Après avoir participé à de telles initiatives, les jeunes mères présentent des taux de soutien perçu plus élevés, des taux de dépression inférieurs, un sentiment de compétence parentale et une estime de soi supérieurs. Certaines études ayant utilisé l'observation dyadique comme méthodologie démontrent également que l'apport de soutien à la jeune mère améliore la qualité des interactions qu'elle entretient avec son enfant (Clewell et al., 1989 ; Jacobson et Frye, 1991). Dans cette perspective, cette approche atteint non seulement des objectifs de soutien mais également certains objectifs indirects liés au bien-être de la mère et à l'amélioration de son comportement envers son enfant. Cependant, dans plusieurs recensions importantes sur ces questions, Furstenberg et ses collègues $(1987 ; 1989)$ ainsi que Clewell et al. (1989) et Benasich et al. (1992) suggèrent que l'effet sur le développement de l'enfant est relativement modeste.

\section{Les effets et limites de l'intervention auprès des dyades enfant-mère adolescente}

Plusieurs constatations émanent de cette brève description des interventions auprès des dyades enfant-mère adolescente. Premièrement, ces interventions semblent efficaces dans la mesure où l'évaluation porte sur les objectifs directs. Bien que variable, l'impact positif de l'intervention à l'égard des connaissances développementales, des croyances parentales et des ressources de soutien est indéniable (Benasich et al., 1992 ; Clewell et al., 1989). Cependant, les résultats à l'égard de l'amélioration des comportements maternels envers l'enfant, un des objectifs indirects des modèles d'intervention, demeurent relativement modestes (Osofsky et al., 1988 ; 1993) et l'impact de ces stratégies sur la qualité du développement émotionnel et social de l'enfant reste assez faible (Furstenberg et al., 1989). 
Deuxièmement, ces stratégies reflètent l'état des connaissances concernant les facteurs contextuels qui caractérisent l'écologie sociale des dyades enfant-mère adolescente. Ces stratégies reposent sur l'idée que si l'on modifie certaines caractéristiques contextuelles de la dyade (soutien social) et personnelles de la mère (connaissances développementales), les processus dyadiques et familiaux qui ont le potentiel d'influencer le développement de l'enfant seront aussi influencés (Chamberland, 1996). Or, les résultats des évaluations des stratégies d'intervention ne confirment pas cette hypothèse. Certes, il est pertinent d'influencer le contexte de la dyade et le bien-être de la mère et, à cet égard, les interventions décrites semblent avoir un effet favorable. Cependant, ces travaux indiquent que cet effet se répercute trop peu souvent sur l'enfant (Osofsky et al., 1993). Lorsque l'on considère que le risque psychosocial des enfants de jeunes mères se concrétise en problèmes vécus surtout au niveau du développement socio-affectif, la difficulté d'influencer les enfants par l'intervention devient un problème nécessitant une remise en question des principes de base de l'intervention (Lamb, 1988).

Troisièmement, Osofsky et ses collègues (1993) et Aber (1992) soulignent que dans la majorité des cas, l'intervention auprès des jeunes mères et de leur enfant $n$ 'est fondée sur aucune prémisse théorique ayant une validité empirique. À cause du fait que les stratégies d'intervention portent surtout sur les facteurs structuraux, contextuels ou personnels, certaines questions ne sont pas abordées. Par exemple, quels aspects de la dyade mère-enfant doivent être abordés afin que le développement de l'enfant en soit directement influencé ? De quelle façon la stratégie d'intervention doit-elle être modifiée pour faire face aux diverses problématiques développementales (Krupka, 1996) ? Enfin, il est nécessaire de considérer qu'au niveau du développement socio-affectif de leur enfant et de leurs antécédents familiaux, les jeunes mères se distinguent des autres groupes défavorisés (Aber, 1992). De plus, la recherche dans ces domaines nous montrent que les jeunes mères représentent un groupe encore plus diversifié que celui des clientèles provenant de contextes non à risque (Ward et Carlson, 1995). Il est pertinent de concevoir la problématique des dyades enfant-mère adolescente d'une manière permettant de mieux cerner le développement socio-affectif de l'enfant en plus du bien-être de la jeune mère et de tenir compte de la diversité des dyades et des problématiques qui s'y développent (Aber, 1992).

\section{UNE PERSPECTIVE RELATIONNELLE DES DIFFICULTÉS ENFANT-MÈRE ADOLESCENTE}

Nous proposons qu'au-delà des conditions de vie des dyades enfantmère adolescente, il est pertinent de considérer le vécu relationnel des jeunes mères. Les processus dyadiques interactionnels et relationnels parent-enfant 
sont décrits comme étant parmi les moteurs du développement socio-affectif de l'enfant. Ces processus représentent le mécanisme par lequel se transmettent les conditions contextuelles et familiales à l'enfant (Belsky, 1993 ; Bronfenbrenner, 1996). Bronfenbrenner souligne que dans l'écologie développementale de l'enfant, les variables qui ne le concernent pas directement (p. ex., pauvreté, scolarité maternelle) exercent un impact sur lui principalement par la façon dont elles influencent la qualité des interactions parent-enfant. De même, Belsky, reconnaissant d'emblée que le développement de l'enfant dans les contextes à risque est déterminé par de multiples facteurs, démontre que même dans des conditions défavorables, lorsque les parents sont en mesure de répondre adéquatement aux besoins affectifs de leur enfant, le développement de ce dernier n'est pas compromis. Il faut également considérer que dans un contexte d'adversité comme celui que connaissent les jeunes mères, des antécédents relationnels positifs peuvent aider dans le développement d'un réseau de soutien et au niveau de leur bienêtre. Selon Aber (1992), un des défis de la recherche et de l'intervention auprès des jeunes mères et de leur enfant concerne l'intégration de leur vécu relationnel dans la compréhension de leurs difficultés, ces éléments étant relativement absents des modèles actuels.

Nous proposons d'approfondir cette dimension de l'écologie développementale de la dyade enfant-mère adolescente en deux étapes. D'abord nous décrirons les relations des jeunes mères : celles qu'elles ont vécues dans le passé, celles qu'elles entretiennent aujourd'hui avec leurs parents et leurs pairs, et celles qu'elles établissent avec leur enfant. Ensuite, nous situerons la question relationnelle à l'intérieur des prémisses, des procédures et des résultats empiriques afférents à la théorie de l'attachement (Ainsworth, Blehar, Waters et Wall, 1978 ; Bowlby, 1969). Les chercheurs dans ce domaine continuent d'établir la validité de cette perspective sur les plans empirique et clinique (Carlson et Sroufe, 1995), la rendant utile dans l'intégration et la compréhension des travaux sur les relations des jeunes mères et de leur enfant.

\section{Les relations antécédentes}

Sur le plan de leur propre histoire développementale, les mères adolescentes sont souvent elles-mêmes les enfants de parents adolescents, indiquant qu'elles ont elles aussi été exposées à des conditions de vie et à un contexte relationnel possiblement difficiles (Lamb, 1988). Dans $60 \%$ des cas, les jeunes mères rapportent avoir vécu des expériences sexuelles coercitives, de l'abus sexuel ou de la maltraitance de la part d'un membre de leur famille, comparativement à un taux de $10 \%$ dans la population générale (Osofsky et al., 1988). Lorsque les jeunes mères quittent la maison, les relations qu'elles 
entretiennent avec leurs parents peuvent demeurer conflictuelles et devenir des stresseurs, plutôt que des sources de soutien dans la période de la transition à la parentalité (Spieker et Bensley, 1994).

\section{Les relations actuelles}

Les relations que les jeunes mères entretiennent avec les autres personnes de leur milieu - leurs pairs, leur conjoint — sont également problématiques. L'isolement et le peu de soutien social des jeunes mères témoignent des difficultés relationnelles qu'elles vivent auprès des personnes de leur entourage. De plus, la présence du père de l'enfant n'est pas nécessairement une source de soutien (Spieker et Bensley, 1994) et les relations avec les partenaires sont souvent conflictuelles, ayant pour résultat que les jeunes mères changent régulièrement de conjoint. Dans une recherche souvent citée dans la littérature, Pianta et Egeland (1990) démontrent que le changement fréquent de conjoint constitue la variable contextuelle qui prédit le mieux le degré de sensibilité des jeunes mères envers leur enfant.

\section{Les relations avec l'enfant}

Les relations dysfonctionnelles vécues par la jeune mère s'étendent à la relation qu'elle établit avec son enfant. Les interactions enfant-mère adolescente se caractérisent par des niveaux excessifs d'interférence et de coercition de la part de la mère, par l'usage fréquent d'interventions physiques, par de l'incohérence et de l'imprévisibilité dans les réponses maternelles, ainsi que par le rejet manifeste de l'enfant dans de nombreux cas (Ward et Carlson, 1995). De plus, les mères adolescentes parlent peu à leur enfant, ne leur portant pas d'attention pendant des périodes prolongées. De même, elles se retrouvent rarement dans des échanges affectifs avec leur enfant (Krupka, 1996). Crittenden (1988) qualifie ces types d'interactions de « relations à risque ", liées aux problèmes d'ajustement chez l'enfant.

Parfois, les relations des jeunes mères avec leur enfant sont à l'extrême de la dysfonctionalité et deviennent abusives. À cet égard, les enfants des jeunes mères sont parmi les plus susceptibles de faire l'objet d'un signalement aux organismes de protection de la jeunesse. Tandis que Bolton (1990) et Dukewich et al. (1996) qualifient l'âge de la mère comme l'un des facteurs de risque les plus importants dans la prédiction de la maltraitance de l'enfant, d'autres études confirment qu'environ $20 \%$ des enfants des jeunes mères se retrouvent dans des conditions d'abus ou de négligence (Dukewich et al., 1996). Bolton estime qu'entre 36 et $51 \%$ des signalements aux organismes de protection aux États-Unis impliquent des enfants de mères adolescentes. 
Par le biais de cette brève description du vécu relationnel des jeunes mères et des relations qu'elles établissent avec leur enfant, nous proposons que la problématique des jeunes mères et le risque psychosocial peuvent être perçus dans une perspective relationnelle, plutôt qu'uniquement au niveau des facteurs contextuels. Notamment, ces recherches suggèrent qu'une des variables qui distingue les dyades enfant-mère adolescente des autres dyades à risque concerne leur exposition régulière à des relations dysfonctionnelles (Aber, 1992). En développant des stratégies d'intervention ayant pour objectif direct l'amélioration de la relation parent-enfant, on touche aux mécanismes par lesquels le vécu relationnel de la jeune mère et les caractéristiques défavorables de son contexte de vie se transmettent à l'enfant et le rendent à risque de développer des problèmes d'ajustement sur le plan socio-affectif (Belsky, 1993). Il faut préciser qu'une approche relationnelle ne diminue pas l'importance des facteurs structuraux et personnels, mais plutôt les conçoit comme qualifiant le contexte dans lequel s'élabore la relation.

La simple description des relations enfant-mère adolescente comme n'étant pas favorables ne nous permet pas d'atteindre l'objectif de décrire les différents cadres relationnels des dyades enfant-mère adolescente. Dans une série de travaux, Osofsky et ses collègues $(1988 ; 1993)$ rapportent les résultats d'interventions centrées sur les besoins relationnels des dyades enfant-mère adolescente. Malgré le fait que ce programme a obtenu un certain succès, l'évaluation fut non concluante en ce qui concerne l'amélioration du développement socio-affectif de l'enfant. Parmi les raisons données par les auteurs pour expliquer cet insuccès, une des plus importantes concerne le fait que les programmes sont appliqués sans tenir compte des thématiques cliniques qui se présentent à l'intervenant. Selon ces auteurs et d'autres (Ward et Carlson, 1995), il est nécessaire de pouvoir distinguer entre les profils relationnels qui caractérisent les dyades enfant-mère adolescente afin de pouvoir ajuster l'intervention. C'est dans cette perspective que nous abordons l'attachement parent-enfant.

\section{LA PERTINENCE DE LA THÉORIE DE L'ATTACHEMENT}

Les avantages de la théorie de l'attachement pour l'élaboration d'une approche relationnelle à l'intervention auprès des dyades enfant-mère adolescente se situent à deux niveaux. Premièrement, la théorie, les concepts et les méthodes liés à l'attachement parent-enfant tel que défini par Bowlby (1969) et Ainsworth et al., (1978) ont été validés de manière importante au cours des 30 dernières années. Le développement et la validation de la «Situation Étrangère » (SE), principale mesure de l'attachement en petite enfance (Ainsworth et al., 1978), ainsi que ceux d'autres mesures d'interaction parent-enfant et de compétence parentale assurent à notre démarche 
une assise scientifique en ce qui concerne les concepts adoptés pour l'intervention et les protocoles d'évaluations (voir De Wolff et van IJzendoorn, 1997). Deuxièmement, la recherche sur l'attachement a permis l'identification de quatre modèles relationnels majeurs pouvant être utilisés afin de distinguer le cheminement relationnel d'une dyade permettant d'ajuster l'intervention (Ainsworth et al., 1978; Main et Solomon, 1990). Les mères qui développent des relations d'attachement sécurisantes avec leur enfant sont sensibles à ses besoins, et répondent de façon cohérente et appropriée. Les interactions mère-enfant revêtent un caractère soutenant et structurant pour faciliter le jeu et l'exploration de l'enfant. Dans les relations évitantes, le comportement des mères lors d'interactions se décrit comme prévisiblement intrusif, interférant ou rejetant, surtout lorsque l'enfant démontre de la vulnérabilité émotionnelle, l'enfant étant valorisé pour la performance cognitive et le contrôle de son état affectif (Pederson et Moran, 1996). Dans les relations ambivalentes, les interactions mère-enfant se distinguent par leur manque de prévisibilité et de cohérence, les mères étant parfois sensibles aux besoins et aux comportements de l'enfant, mais leurs réponses reflètant une mauvaise perception des signaux. L'enfant demeure préoccupé par la proximité de son parent et devient facilement en détresse. Ces comportements semblent avoir pour but d'augmenter la probabilité de réponse de la part du parent dans des situations de danger (Cassidy et Berlin, 1994).

Les relations désorganisées sont peu connues, mais semblent un mélange extrême des relations évitantes et ambivalentes. On retrouve ces relations surtout dans les milieux où il y a abus. L'enfant oscille entre des stratégies d'évitement (tentative de gérer lui-même sa détresse) et d'ambivalence (résistance à l'interaction, mais recherche de proximité) dans les interactions avec son parent. De plus, on conçoit habituellement qu'une relation désorganisée marque les prémices d'une des autres relations insécurisantes, soit évitante ou ambivalente, aidant à qualifier davantage la relation. Les chercheurs dans le domaine de l'attachement stipulent que l'attribution de relation désorganisée, avec une désignation secondaire évitante ou ambivalente comporte un risque développemental plus élevé qu'une simple désignation de relation insécurisante (Carlson et Sroufe, 1995).

La description de ces modèles relationnels, identifiés dans le contexte des travaux sur l'attachement et validés sur le plan empirique, suggèrent que cette perspective peut bien se prêter au développement d'une stratégie d'intervention auprès des dyades enfant-mère adolescente orientée sur la question relationnelle. 


\section{DESCRIPTION D'UNE STRATÉGIE D'INTERVENTION POUR LES DYADES ENFANT-MÈRE ADOLESCENTE}

Dans cette section, nous voulons décrire une stratégie d'intervention à l'intention des dyades enfant-mère adolescente que nous expérimentons dans le contexte du programme de recherche « Être Parent ». Trois aspects sont décrits : 1) les détails de l'administration de l'intervention; 2) les caractéristiques des rencontres d'intervention; 3) la stratégie d'évaluation de l'intervention.

\section{Détails de l'administration de l'intervention}

L'objectif direct de l'intervention est d'améliorer la relation mère-enfant afin d'avoir un impact sur le développement socio-affectif de l'enfant. L'intervention commence lorsque l'enfant est âgé de 6 mois. Cet âge a été choisi pour deux raisons principales. D'abord parce que certains écrits démontrent que c'est vers cet âge que l'enfant est davantage impliqué sur les plans cognitif et moteur dans les interactions sociales, ce qui augmente la probabilité que des changements dans le comportement maternel ait un impact sur lui (Cohn, Campbell et Ross, 1991). Ensuite, les résultats de Krupka (1996) concernant le stress et la dépression indiquent qu'avant 6 mois, les jeunes mères sont souvent entourées de leurs amis et que le besoin d'aide ne s'exprime pas de façon manifeste. À 6 mois, le réseau de la jeune mère s'effrite et elle se retrouve seule, présentant des taux de stress et de dépression élevés. À ce moment, il est plus envisageable que la jeune mère soit réceptive à la possibilité de participer à l'intervention que nous proposons.

L'intervention comprend douze visites à domicile hebdomadaires lorsque l'enfant a entre 6 et 10 mois. La première visite, effectuée par deux personnes formées au préalable, consiste en une évaluation semi-structurée du développement de l'enfant, des comportements maternels et de la relation mère-enfant. D'autres informations sont également recueillies (p. ex., conditions de vie, soutien social, perceptions de l'enfant, stress, dépression). La visite comprend une entrevue générale, portant sur les caractéristiques de la grossesse, de l'accouchement, du retour à la maison et sur les activités quotidiennes de la dyade. Ensuite, une évaluation du développement moteur et mental de l'enfant est réalisée. Une séquence de jeu libre est ensuite enregistrée sur bande vidéo et finalement, on demande à la jeune mère de compléter des questionnaires. Nous profitons du contexte d'évaluation pour obtenir de manière indirecte des informations d'ordre relationnel. Pendant la visite d'évaluation (120 minutes), nous observons les comportements de l'enfant et de la mère afin d'obtenir un portrait des interactions dyadiques. Pederson et Moran (1996) ont démontré que le fait de placer certaines demandes sur la dyade fait ressortir des éléments essentiels de la relation. 
Nous pouvons ainsi observer comment la mère répond aux besoins et signaux de son enfant, ainsi que voir la façon dont son enfant organise ses comportements autour d'elle.

Après la visite d'évaluation, les observateurs complètent le tri-de-carte de sensibilité maternelle (Pederson et Moran, 1996), donnant une description générale des comportements maternels qui ont été observés. Ensuite, les évaluateurs et une troisième personne se rencontrent afin de créer une description narrative de la visite. Le but de cette rencontre est d'identifer le type de relation qui se développe afin de mieux cibler la stratégie à employer durant l'intervention. Diverses questions précises sont abordées durant l'entrevue et la période des questionnaires : Est-ce que la mère prend connaissance des comportements et signaux de son enfant ? Est-ce qu'elle semble ignorer son enfant pendant des périodes prolongées (p. ex., bébé placé dans une chaise haute seul pendant 45 minutes) ? Est-ce qu'il y a des échanges chaleureux entre la mère et le bébé durant l'évaluation ? Est-ce que la mère taquine le bébé avec les jouets (p. ex., placer un jouet à proximité et lorsque le bébé l'atteint, le lui prendre et le placer plus loin) ?

On pose également des questions concernant l'enfant : Durant la période d'évaluation motrice et mentale, est-ce que le bébé demeure concentré sur la tâche ? Est-ce que l'état émotionnel de l'enfant est prévisiblement lié aux interactions avec sa mère ? Y a-t-il des crises ou des sautes d'humeur difficiles à expliquer ? La description de la visite aide les évaluateurs à savoir s'il s'agit d'une relation sécurisante, évitante ou ambivalente (ou désorganisée avec l'une des autres relations en sous-catégorie).

En plus d'identifier le type de relation, l'intervenante doit situer le comportement de la mère dans le contexte de la perspective de l'attachement. Si la jeune mère est sensible, répondant de façon chaleureuse et prévisible à l'enfant, l'intervention aura pour objectif de maintenir ses acquis. Si la jeune mère dénigre, ignore ou rejette son enfant, ou encore, si elle recherche la compétence de l'enfant au détriment de son état affectif, l'objectif de l'intervention sera de la sensibiliser au fait que son enfant expérimente des émotions durant ses interactions avec elle et que les comportements et le développement de son enfant sont influencés par ses actions. S'il s'agit d'une dyade dans laquelle la jeune mère montre des signes de sensibilité à l'égard de son enfant, mais que ses comportements manquent de régularité, le but premier de l'intervention sera d'offrir le soutien nécessaire afin de rendre le comportement maternel plus constant.

\section{Caractéristiques des rencontres d'intervention}

L'intervention, tirée et adaptée de Krupka (1996), est présentée aux jeunes mères (14 à 19 ans) comme un atelier de stimulation de l'enfant 
s'effectuant à domicile. Elle comprend quatre objectifs principaux, dont l'importance varie selon le type de relation qui a été identifiée durant l'évaluation initiale. Ces objectifs sont : 1) soutenir et renforcer les compétences parentales déjà présentes chez la jeune mère ; 2) sensibiliser la jeune mère à la façon dont ses actes influencent l'état émotionnel et le développement de son enfant ; 3) sensibiliser la jeune mère à la signification des divers signaux et manifestations affectives de l'enfant ; et 4) établir une première histoire positive d'interactions pour la mère et l'enfant.

De façon générale, chaque visite est divisée en trois parties. Durant la première partie, l'intervenante cherche à établir un contact, un rapport de confiance avec la jeune mère. Durant ces moments, l'intervenante prend note des besoins manifestés par l'adolescente en termes de soutien émotionnel et instrumental et tente de mieux cerner ses conditions de vie aux plans financier, social, conjugal, ainsi que la façon dont la jeune mère se représente son enfant et son rôle de parent.

La seconde partie consiste en un enregistrement vidéo d'une interaction mère-enfant d'environ 5-7 minutes. Cette séquence sert d'outil pédagogique afin d'identifier auprès de la mère les moments où l'enfant lui signale un besoin (p. ex., l'enfant vocalise ou tend les bras), de renforcer auprès de la jeune mère ses comportements positifs, répondant à des comportements de son enfant ou montrant un degré de sensibilité (p. ex., " ton bébé a arrêté de pleurer tout de suite quand tu l'as pris dans tes bras ») et de valoriser les interactions mère-enfant où il semble y avoir un degré de cohérence et d'affect partagé entre les deux (p. ex., « as-tu vu comment ton bébé te regardait quand tu lui parlais doucement ? »).

Dans la troisième partie, l'intervenante prend un rôle plus directif en incitant la jeune mère à participer à des activités d'interaction précises avec son enfant (p. ex., imitation, procédure du visage impassible). Le but de ces activités est de donner un contexte et une occasion à la jeune mère de détecter les signaux et comportements de son enfant, d'agir de façon cohérente et prévisible et de lui permettre de remarquer les comportements de son enfant lorsqu'elle agit de manière sensible. Ces activités favorisent la réciprocité des interactions, l'état émotionnel de l'enfant et la compréhension des comportements de l'enfant par la mère.

Après chaque visite, l'intervenante doit effectuer une évaluation des comportements maternels qu'elle a pu observer, en utilisant les échelles d'Ainsworth (Ainsworth et al., 1978) et celles rapportées par Krupka (1996), afin de déterminer si des changements dans la stratégie d'intervention sont nécessaires. 


\section{Évaluation de l'intervention}

En élaborant ce projet, nous voulions porter une attention particulière à la question de l'évaluation. La plupart des interventions auprès des jeunes mères ne sont pas évaluées ou portent uniquement sur le bien-être des mères, sur ses connaissances développementales ou sur ses attitudes parentales, rarement sur les interactions ou le développement socio-affectif de l'enfant (Clewell et al., 1989). De plus, à notre connaissance, aucune étude publiée ne rapporte un suivi longitudinal d'une intervention auprès de dyades enfant mère-adolescente, les effets rapportés portant uniquement sur la période qui suit immédiatement la fin de l'intervention. Dans l'évaluation de l'intervention, nous voulions donc considérer le bien-être de la jeune mère, ainsi que ses idées concernant le développement de l'enfant, mais nous voulions aussi apporter une attention particulière aux variables reliées aux interactions mèreenfant.

Quatre-vingt dyades enfant-mère adolescente participent à notre recherche, ainsi que quarante dyades enfant-mère adulte. Les 80 jeunes mères sont assignées de façon randomisée à un groupe d'intervention ou à un groupe de comparaison. Les membres du groupe de comparaison reçoivent les interventions disponibles dans leur communautés. Nous proposons d'examiner un groupe de mères adultes afin de pouvoir mieux évaluer comment le groupe d'intervention se comporte.

Les évaluations pour tous ces groupes sont identiques, toutes les dyades étant suivies lorsque l'enfant est âgé de 6, 10, 15 et 18 mois. Le bien-être des mères est évalué par le biais de mesures autorapportées et de questionnaires portant sur les phénomènes de dépression maternelle, de stress parental, de soutien social, chacun de ces concepts étant mesuré à tous les temps de l'étude. La question des comportements maternels et de la qualité des interactions est abordée par le biais de la mesure de la sensibilité maternelle, lors de la procédure d'observation à domicile décrite dans la section précédente. De plus, d'autres séquences d'interaction fourniront des informations supplémentaires sur les processus proximaux que nous voulons modifier. Enfin, le cceur de l'évaluation du développement de l'enfant comprend des SE (Situations Étrangères) effectuées lors de visites en laboratoire quand les enfants seront âgés de 15 et 18 mois. Bien que nous espérions effectuer des changements au niveau des variables correspondant aux autres mesures dépendantes, nos hypothèses centrales de recherche concernent davantage le comportement maternel et l'attachement à la mère. Plus précisément, nous prévoyons rehausser le niveau de sensibilité maternelle observé à la maison et nous nous attendons à ce que la proportion d'attachement sécurisant dans le groupe d'intervention soit plus élevée que celle dans le groupe de comparaison. 
Puisque l'intervention a lieu entre les deux premières évaluations, nous serons en mesure de documenter s'il y a effet immédiat à 10 mois, de même que durant les deux évaluations suivantes, ce qui nous permettra ainsi d'examiner la stabilité de l'intervention à moyen terme. Il faut noter que, bien que nous nous attendions à un effet « intervention " pour la sensibilité maternelle et l'attachement, nous nous attendons également à une diminution de cet effet à 18 mois. Les changements d'effets à 18 mois nous aideront à mieux cerner les facteurs qui maintiennent les acquis de l'intervention.

Nous avons proposé que, pour toucher plus directement le développement socio-affectif des enfants de jeunes mères, il est nécessaire de modifier la relation mère-enfant de manière précoce. La mesure de succès que nous aurons dans l'atteinte de cet objectif nous informera tant sur le rôle de cette relation dans le développement que sur l'efficacité de notre intervention.

\section{BIBLIOGRAPHIE}

ABER, J. L. (1992), “ Comments on "A model of adolescent parenting" ", Human Development, vol. 35, p. 100-107.

AINSWORTH, M. D., BLEHAR, M., WATERS, E. et WALL, S. (1978), Patterns of attachment : A study of the strange situation, Hillsdale, NJ, Erlbaum.

BELSKY, J. (1993), « Etiology of Child Maftreatment : A developmental-ecological analysis ", Psychological Bulletin, vol. 114, p. 413-434.

BENASICH, A. A., BROOKS-GUNN, J. et CLEWELL, B. C. (1992), « How do mothers benefit from early intervention? ", Journal of Applied Developmental Psychology, vol. 13, p. 311-362.

BOLTON, F. G. (1990), « The risk of child maltreatment in adolescent parenting ", Advances in Adolescent Mental Health, vol. 4, p. 223-227.

BOWLBY, J. (1969), Attachment and Loss, Vol. 1 : Attachment, New York, Basic Books.

BROOKS-GUNN, J. et FURSTENBERG, F. F. (1986), "The children of adolescent mothers : Physical, academic and psychological outcomes ", Developmental Review, vol. 6, p. 224-251.

BROOKS-GUNN, J. et FURSTENBERG, F. F. (1987), "Continuity and change in the context of poverty : Adolescent mothers and their children », in GALLAGHER, J. J. (dir.), The Malleability of Children, (p. 171-188), Baltimore, MD, Brooks.

BRONFENBRENNER (1996), « Le Modèle de recherche Processus-Personne-Contexte dans la recherche en développement », in TESSIER, R. et TARABULSY, G. M. (dir.), Le Modèle écologique dans l'étude du développement de l'enfant, (p. 9-59), Sainte Foy, Les Presses de l'Université du Québec.

CARLSON, E. A. et SROUFE, L. A. (1995), « Contribution of attachment theory to developmental psychopathology , in CICCETTI, D. et COHEN, D. J. (dir.), Developmental Psychopathology, Vol. $1:$ Theory and Methods, (p. 581-617), New York, Wiley.

CASSIDY, J. et BERLIN, L. J. (1994), "The insecure-ambivalent pattern of attachment ", Child Development, vol. 65, p. 971-991. 
CHAMBERLAND, C. (1996), " Écologie et prévention : Pertinence pour la santé communautaire , in TESSIER, R. et TARABULSY, G. M. (dir.), Le Modèle écologique dans l'étude du développement de l'enfant, (p. 61-79), Sainte Foy, Les Presses de l'Université du Québec.

COHN, J. F., CAMPBELL, S. et ROSS, S. (1991), "Infant response in the still-face paradigm at 6 months predicts avoidant and secure attachment at 12 months", Development and Psychopathology, vol. 3, p. 367-376.

CLEWELL, B. C., BROOKS-GUNN, J. et BENASICH, A. A. (1989), « Evaluation childrelated outcomes of teenage parenting programs ", Family Relations, vol. 38, p. 201209.

CRITTENDEN, P. (1988), « Relationships at risk ", in BELSKY, J. et NEZWORSKY, T., Clinical Implications of Attachment, (p. 136-174), Hillsdale, NJ, Erlbaum.

DEWOLFF, M. S. et VAN IJZENDOORN, M. H. (1997), « Sensitivity and attachment : A meta-analysis on parental antecedents of of infant attachment », Child Development, vol. 68, p. 571-591.

DUKEWICH, T. L., BORKOWSKI, J. G. et WHITMAN, T. L. (1996), « Adolescent mothers and child abuse potential ", Child Abuse et Neglect, vol. 20, p. 1031-1148.

FURSTENBERG, F. F., BROOKS-GUNN, J. et CHASE-LANSDALE, L. (1989), "Teenaged pregnancy and childbearing ", American Psychologist, vol. 44, p. 313-320.

FURSTENBERG, F. F., BROOKS-GUNN, J. et MORGAN, S. P. (1987), Adolescent Mothers in Later Life, Cambridge, England, Cambridge.

JACOBSON, S. et FRYE, K. (1991), «Effect of matemal social support on attachment : Experimental evidence ", Child Development, vol. 62, p. 572-582.

KRUPKA, A. (1996), The Relationship of Early Experience to Development at 13 Months : The Case of Adolescent Mothers and Their Infants, manuscript, University of Westem Ontario.

LAMB, M. E. (1988), « The ecology of adolescent pregnancy and parenthood ", in PENCE, A. R. (dir.), Ecological Research with Children and Families, (p. 99-121), New York, Teachers' College.

MAIN, M. et SOLOMON, J. (1990), " Procedures for identifying infants as disorganized during the strange situation ", in GREENBERG, M., CICCHETTI, D. et CUMMINGS, E. (dir.), Attachment in the Preschool Years, (p. 121-160), Chicago, University of Chicago Press.

OSOFSKY, J. D., CULP, A. M. et WARE, L. M. (1988), «Intervention challenges with adolescent mothers and their infants ", Psychiatry, vol. 51, p. 236-248.

OSOFSKY, J. D., HANN, D. M. et PEEBLES, C. (1993), « Adolescent parenthood : Risks and opportunities for mothers and infants ", in ZEANAH, C. (dir.), Handbook of Infant Mental Health, (p. 106-119), New York, Guilford.

PAGEAU, M., FERLAND, M., CHOINÈRE, R. et SAUVAGEAU, Y. (1997), Indicateurs sociosanitaires : le Québec et ses régions, Gouv. du Québec, Ministère de la Santé et des Services sociaux.

PEDERSON, D. R. et MORAN, G. (1996), « Expressions of the attachment relationship outside of the strange situation ", Child Development, vol. 38, p. 915-927.

PIANTA, R. C. et EGELAND, B. (1990), "Life stress and parenting outcomes in a disadvantaged sample : Results of the mother-child interaction project ", Journal of Clinical Child Psychology, vol. 19, p. 329-336. 
SCHELLENBACH, C. J., WHITMAN, T. L. et BORKOWSKI, J. G. (1992), « Toward an integrative model of adolescent parenting ", Human Development, vol. 35, p. 81-99.

SPIEKER, S. J. et BENSLEY, L. (1994), * Roles of living arrangements and grandmother support in adolescent mothering and infant attachment ", Developmental Psychology, vol. 30, p. 102-111.

TRAD, P. (1994), « Deterring psychopathology in infants of adolescent mothers », International Journal of Adolescent Medecine \& Health, vol. 7, p. 27-63.

TURNER, R. J., GRINDSTAFF, C. F. et PHILLIPS, N. (1990), « Social support and outcome for teenage pregnancy ", Journal of Health and Social Behavior, vol. 31, p. 43-57.

WARD, M. J. et CARLSON, E. A. (1995), « Associations among adult attachment, maternal sensitivity, and attachment in a sample of adolescent mothers ", Child Development, vol. 66, p. $69 \cdot 79$. 